International Journal of Applied Mathematics

Volume 28 No. $5 \quad 2015,593-603$

ISSN: 1311-1728 (printed version); ISSN: 1314-8060 (on-line version)

doi: http://dx.doi.org/10.12732/ijam.v28i5.11

\title{
A FAMILY OF THE ZECKENDORF THEOREM RELATED IDENTITIES
}

\author{
Ivica Martinjak \\ Faculty of Science \\ University of Zagreb \\ Bijenička cesta 32, HR-10000 Zagreb, CROATIA
}

\begin{abstract}
In this paper we present a family of identities for recursive sequences arising from a second order recurrence relation, that gives instances of Zeckendorf representation. We prove these results using a special case of an universal property of the recursive sequences. In particular cases we also establish a direct bijection. Besides, we prove further equalities that provide a representation of the sum of $(r+1)$-st and $(r-1)$-st Fibonacci number as the sum of powers of the golden ratio. Similarly, we show a class of natural numbers represented as the sum of powers of the silver ratio.
\end{abstract}

AMS Subject Classification: 11B39, 11B37

Key Words: Zeckendorf representation, recursive sequence, Fibonacci numbers, Pell numbers, Diophantine equation

\section{Introduction}

According to the Zeckendorf Theorem, every natural number $n$ is uniquely represented as a sum of nonconsecutive Fibonacci numbers $F_{k}$,

$$
n=F_{k_{1}}+F_{k_{2}}+\cdots+F_{k_{m}}, \quad k_{i+1} \geq k_{i}+2, \quad k_{i} \geq 2 .
$$

Such a sum is called the Zeckendorf representation of $n[9,10]$. The Fibonacci sequence can be naturally extended to negative indexes using the same defining recurrence relation, and terms in this sequence are sometimes called negafibonacci numbers. D. Knuth has shown that there is a unique representation of an integer $N$ in negafibonacci numbers, see [7]. Representations 


$$
79=F_{10}+F_{8}+F_{4}=55+21+3
$$

and

$$
-37=F_{-5}+F_{-7}+F_{-10}=5+13+(-55)
$$

are examples of the former and the latter. The Fibonacci identities like

$$
\begin{aligned}
4 F_{n} & =F_{n+2}+F_{n}+F_{n-2}, \quad n \geq 2 \\
5 F_{n} & =F_{n+3}+F_{n-1}+F_{n-4}, \quad n \geq 4 \\
6 F_{n} & =F_{n+3}+F_{n+1}+F_{n-4}, \quad n \geq 4 \\
11 F_{n} & =F_{n+4}+F_{n+2}+F_{n}+F_{n-2}+F_{n-4}, \quad n \geq 4
\end{aligned}
$$

give examples of a Zeckendorf representation. According to (2), we have

$$
\begin{aligned}
65 & =F_{10}+F_{6}+F_{3}=55+8+2, \\
105 & =F_{11}+F_{7}+F_{4}=89+13+3, \ldots
\end{aligned}
$$

Note that the indexes of the Fibonacci numbers within these identities are the same as the exponents in the expansion of natural numbers in powers of the golden ratio $\phi$. In particular, $5=\phi^{3}+\phi^{-1}+\phi^{-4}, 6=\phi^{3}+\phi^{1}+\phi^{-4}, \ldots$

This paper aims at finding identities encountering Zeckendorf representation. We also extend these ideas to more general recursive sequences.

\section{Preliminaries}

Given $c_{1}, c_{2}, \ldots, c_{k} \in \mathbb{N}_{0}$, a $k$-th order linear recurrence is defined by the recurrence relation

$$
a_{n}=c_{1} a_{n-1}+c_{2} a_{n-2}+\cdots+c_{k} a_{n-k}, \quad n \geq k
$$

and the initial values $a_{0}, a_{1}, \ldots, a_{k-1}$. By $\left(a_{n}\right)_{n \geq 0}$ we denote the sequence of numbers $a_{0}, a_{1}, \ldots$ defined by this recurrence. The following lemma gives a combinatorial interpretation of the terms of $\left(a_{n}\right)_{n \geq 0}[2]$.

Lemma 1. Let $a_{0}=1$. Then $a_{n}$ is equal to the number of colored tilings of an $n$-board with tiles of length at most $k$, where a tile of length $i$ can be colored in $c_{i}$ colors, $1 \leq i \leq k$.

Proof. We prove that the number of an $n$-board tilings obey the same recurrence relation as the sequence $\left(a_{n}\right)_{n \geq 0}$. 
By $a_{n}^{\prime}$ we denote the number of tilings of an $n$-board. The set of all such $n$-board tilings can be divided into $k$ subsets, where tilings in the $i$-th subset begin with a tile of length $i, 1 \leq i \leq k$. The number of tilings in the $i$-th subset is equal to $c_{i} a_{n-i}^{\prime}$ which means that the whole set of $n$-board tilings counts $a_{n}^{\prime}$ tilings,

$$
a_{n}^{\prime}=c_{1} a_{n-1}^{\prime}+c_{2} a_{n-2}^{\prime}+\cdots+c_{k} a_{n-k}^{\prime} .
$$

From the fact that $a_{0}=a_{0}^{\prime}=1$ it follows $a_{n}^{\prime}=a_{n}$ which completes the proof.

When $k=2$ relation (5) reduces to a second order recurrence relation,

$$
u_{n+2}=s u_{n+1}+t u_{n}, \quad n \geq 0 .
$$

This class of recurrences is of particular interest. According to previous arguments, when $u_{0}=1$ then $u_{n}$ represents the number of $n$-board tilings with tiles of length 1 and 2, where these tiles are colored in $s$ and $t$ colors, respectively. Tiles of length 1 are called squares while tiles of length 2 are called dominoes.

We use a notion of a breakable cell of a board tiling when proving the following Lemma 2. It is said that an $n$-board tiling is breakable at cell $m$ if it contains a square at cell $m$ or a domino at cells $m-1$ and $m$. Otherwise it contains a domino covering cells $m$ and $m+1$ and such a tiling is unbreakable at cell $m$.

Lemma 2. For the recursive sequence $\left(u_{n}\right)_{n \geq 0}$ and $m \geq 0$ we have

$$
u_{m+n}=u_{m} u_{n}+t u_{m-1} u_{n-1} .
$$

Proof. Let consider condition on breakability at cell $m$ of an $(m+n)$-board. We let $A$ denote the set of $(m+n)$-board tilings breakable at cell $m$. On the other hand, let the set $B$ contains those tilings that are unbreakable at cell $m$. Clearly, the set $A$ counts $u_{m} u_{n}$ elements while the set $B$ counts $t u_{m-1} u_{n-1}$ tilings. The fact that

$$
u_{m+n}=|A|+|B|
$$

completes the statement of the lemma.

In what follows in this paper Lemma 2 has proved to be very useful.

Two notable representatives of the sequences defined by a second order recurrence (6) are the Fibonacci sequence and the Pell sequence. We let $\left(F_{n}\right)_{n \geq 0}$ 
denote the Fibonacci sequence and $\left(P_{n}\right)_{n \geq 0}$ denote the Pell sequence. The Fibonacci sequence arises from (6) when $s=t=1$ and when initial values are 0 and 1. In other words the sequence of Fibonacci numbers is defined by the recurrence

$$
F_{n+2}=F_{n+1}+F_{n}, \quad F_{0}=0, \quad F_{1}=1 .
$$

Close companions of the Fibonacci sequence are Lucas numbers $\left(L_{n}\right)_{n \geq 0}$, that are defined by the same recurrence relation but with initial values $L_{0}=2$ and $L_{1}=1$. It is worth mentioning that these sequences can also be defined as the only solutions $(x, y), x=L_{n}, y=F_{n}$ of the Diophantine equation

$$
x^{2}-5 y^{2}=4(-1)^{n}, \quad n \in \mathbb{N}_{0} .
$$

There are numerous properties and identities known for the Fibonacci sequence. One can find more in a classic reference on this subject [8]. Recall that the closed form for Fibonacci sequence, called Binet formula, is

$$
F_{n}=\frac{\phi^{n}-\bar{\phi}^{n}}{\sqrt{5}}
$$

where $\phi=\frac{1+\sqrt{5}}{2}$ and $\bar{\phi}=\frac{1-\sqrt{5}}{2}$ are solutions of the equation $x^{2}-x-1=0$. The golden ratio $\phi$ and its conjugate $\bar{\phi}$ are also solutions of the equation $x^{n}=$ $x^{n-1}+x^{n-2}$ meaning that both values $\phi$ and $\bar{\phi}$ satisfy the Fibonacci recursion $(8)$,

$$
\begin{gathered}
\phi^{n}=\phi^{n-1}+\phi^{n-2} \\
\bar{\phi}^{n}=\bar{\phi}^{n-1}+\bar{\phi}^{n-2} .
\end{gathered}
$$

There is also recurrence relation encountering the $n$-th power of golden ratio and $n$-th Fibonacci number,

$$
\phi^{n}=\phi F_{n}+F_{n-1} .
$$

The Pell sequence is defined by the recurrence relation

$$
P_{n+2}=P_{n+1}+P_{n}, \quad P_{0}=0, \quad P_{1}=1 .
$$

The sequence arising from the same recurrence but with initial values 2 and 1 is called Pell-Lucas sequence. We let $\left(Q_{n}\right)_{n \geq 0}$ denote this sequence. Equivalently, 
the Pell and Pell-Lucas sequence can be defined as the solutions $(x, y), x=$ $Q_{n} / 2, y=P_{n}$ of the Diophantine equations

$$
\begin{aligned}
x^{2}-d y^{2} & =1 \\
x^{2}-d y^{2} & =-1
\end{aligned}
$$

when $d=2$. We let $\varphi$ denote the silver ratio, $\varphi=1+\sqrt{2}$, and we let $\bar{\varphi}=1-\sqrt{2}$. Then the closed formula for the $n$-th term in Pell sequence $P_{n}$ can be written as

$$
P_{n}=\frac{\varphi^{n}-\bar{\varphi}^{n}}{\varphi-\bar{\varphi}}
$$

According to the previous arguments, both the Fibonacci and the Pell sequences can be represented as the number of board tilings. However, terms of these sequences start by 0 which means that they are shifted by 1 in comparison to the sequence of related tilings. More precisely, denoting the number of an $n$-board tilings with uncolored squares and dominoes by $f_{n}$, we have

$$
f_{n}=F_{n+1} .
$$

Similarly, we let $p_{n}$ denote the number of tilings of an $n$-board with squares in two colors and uncolored dominoes. The number of such $n$-board tilings is equal to the $(n+1)$-st Pell number,

$$
p_{n}=P_{n+1} .
$$

It is worth mentioning that there are numerous results known about the Pell sequence, including basic identities presented in [1] and some number properties shown in [3] and [4]. On the Zeckendorf representation by Pell numbers, one can find more in [5] and [6].

\section{The Main Result}

We define the sequence $\left(U_{n}\right)_{n \geq 0}$ such that

$$
u_{n}=U_{n+1} .
$$

Theorem 1. For the sequence $\left(U_{n}\right)_{n \geq 0}, r \in \mathbb{N}, r \equiv 0(\bmod 2)$ and $t=1$

$$
\left(U_{r+1}+U_{r-1}\right) U_{n}=U_{n+r}+U_{n-r} .
$$


Proof. The definition of the sequence $\left(U_{n}\right)_{n \geq 0}$ can be extended to negative indexes. It is obvious that the term having index $-n$ is uniquely determined by that having index $n$,

$$
U_{-n}=(-1)^{n+1} \frac{U_{n}}{t^{n}}
$$

Now we apply Lemma 2 to both terms in the sum $U_{n+r}+U_{n-r}$,

$$
\begin{aligned}
U_{n+r}+U_{n-r} & =t U_{n-1} U_{r}+U_{n} U_{r+1}+t U_{n-1} U_{-r}+U_{n} U_{-r+1} \\
& =t U_{n-1} P_{r}+U_{n} U_{r+1}+(-1)^{r+1} \frac{U_{n-1} U_{r}}{t^{n-1}}+(-1)^{r} \frac{U_{n} U_{r-1}}{t^{n}} \\
& =U_{n} U_{r+1}+(-1)^{r} \frac{U_{n} U_{r-1}}{t^{n}}+U_{n-1} U_{r}\left(t+\frac{(-1)^{r+1}}{t^{n-1}}\right) .
\end{aligned}
$$

When $t=1$ and $r$ is even the expression above reduces to the first two terms, $U_{n} U_{r+1}+U_{n} U_{r-1}$, which completes the proof.

Since both Fibonacci and Pell sequences satisfy constraint on the coefficient $t$ in Theorem 1 there are two immediate corollaries of Theorem 1.

Corollary 1. For the sequence $\left(F_{n}\right)_{n \geq 0}$ of Fibonacci numbers and an even $r \geq 2$ we have

$$
\left(F_{r+1}+F_{r-1}\right) F_{n}=F_{n+r}+F_{n-r}
$$

The first particular representative of the family of identities (16) is

$$
3 F_{n}=F_{n+2}+F_{n-2}
$$

while further identities are

$$
\begin{aligned}
7 F_{n} & =F_{n+4}+F_{n-4} \\
18 F_{n} & =F_{n+6}+F_{n-6} .
\end{aligned}
$$

Corollary 2. For the sequence $\left(P_{n}\right)_{n \geq 0}$ of Pell numbers and an even $r \geq 2$ we have

$$
\left(P_{r+1}+P_{r-1}\right) P_{n}=P_{n+r}+P_{n-r}
$$


Here we have

$$
\begin{aligned}
6 P_{n} & =P_{n+2}+P_{n-2} \\
34 P_{n} & =P_{n+4}+P_{n-4} \\
198 P_{n} & =P_{n+6}+P_{n-6} .
\end{aligned}
$$

as the first three particular identities of the family (20).

Thus, the first representative of (15) arises when $r=2$,

$$
\left(U_{3}+U_{1}\right) U_{n}=U_{n+2}+U_{n-2} .
$$

Note that it also can be proved directly using Lemma 2,

$$
\begin{aligned}
U_{n+2}+U_{n-2} & =U_{n-1} U_{2}+U_{n} U_{3}+U_{n-1} U_{-2}+U_{n} U_{-1} \\
& =U_{n}\left(U_{3}+U_{1}\right) .
\end{aligned}
$$

Moreover, there is a combinatorial proof of this identity and we are going to present it on the instance of Pell sequence. According to the previous definition there are $p_{n}$ ways to tile an $n$-board with squares in two colors and uncolored dominoes. In order to form $(n+2)$-board tilings ending with

i) a domino,

ii) two black squares,

iii) black square and white square, respectively,

iv) white square and black square, respectively,

v) two white squares,

we need five sets of an $n$-board tilings. These $(n+2)$-board tilings are obtained by gluing tiles declared above to the end of $n$-boards in every of these five sets. To complete the set of $(n+2)$-board tilings we need those tilings ending with a square preceded by a domino. This is achieved when we get a set of an $n$-board tilings and insert a domino before a square when appropriate and cut the last domino otherwise. This operation completes the set of $(n+2)$-board tilings and in the same time leave the set of $(n-2)$-board tilings. Clearly, the described procedure of gluing and cut tiles holds in both directions which proves that

$$
6 p_{n}=p_{n+2}+p_{n-2} .
$$


Note that the parameter $r$ within identities (17) - (19) is the same as exponents in the expansion of resulting sum $F_{r+1}+F_{r-1}$ in powers of $\phi$,

$$
3=\phi^{2}+\phi^{-2}, \ldots
$$

Similarly, we have

$$
\begin{aligned}
6 & =\varphi^{2}+\varphi^{-2}, \\
34 & =\varphi^{4}+\varphi^{-4}, \ldots
\end{aligned}
$$

where exponents corresponds with the value of parameter $r$ within identities (21) - (23). These facts are generalized in the following Theorem 2 and Theorem 3.

Theorem 2. For the Fibonacci sequence $\left(F_{n}\right)_{n \geq 0}$ and $r \in \mathbb{Z}, r \equiv 0$ $(\bmod 2)$

$$
F_{r+1}+F_{r-1}=\phi^{r}+\phi^{-r} .
$$

Proof. Applying the closed formula for Fibonacci sequence to (16) we get

$$
\begin{aligned}
& {\left[\frac{\phi^{r+1}-\bar{\phi}^{r+1}}{\sqrt{5}}+\frac{\phi^{r-1}-\bar{\phi}^{r-1}}{\sqrt{5}}\right]\left(\frac{\phi^{n}-\bar{\phi}^{n}}{\sqrt{5}}\right)} \\
& =\frac{\phi^{n+r}-\bar{\phi}^{n+r}}{\sqrt{5}}+\frac{\phi^{n-r}-\bar{\phi}^{n-r}}{\sqrt{5}}\left[\frac{\phi^{r+1}-\bar{\phi}^{r+1}}{\sqrt{5}}+\frac{\phi^{r-1}-\bar{\phi}^{r-1}}{\sqrt{5}}\right] \\
& =\frac{\phi^{n+r}-\bar{\phi}^{n+r}+\phi^{n-r}-\bar{\phi}^{n-r}}{\phi^{n}-\bar{\phi}^{n}} .
\end{aligned}
$$

Now we have to show that equality

$$
\frac{\phi^{n+r}-\bar{\phi}^{n+r}+\phi^{n-r}-\bar{\phi}^{n-r}}{\phi^{n}-\bar{\phi}^{n}}=\phi^{r}+\phi^{-r}
$$

holds true. The l.h.s. and r.h.s. of this relation reduce immediately to

$$
\begin{aligned}
-\bar{\phi}^{n+r}-\bar{\phi}^{n-r} & =-\phi^{r} \bar{\phi}^{n}-\bar{\phi}^{n} \phi^{-r} \\
\bar{\phi}^{r}+\bar{\phi}^{-r} & =\phi^{r}+\phi^{-r} .
\end{aligned}
$$

Finally, we use a property

$$
-\frac{1}{\phi}=\bar{\phi}
$$


of the golden ratio and its conjugate. For even $r$ we have

$$
\frac{1}{\phi^{r}}=\bar{\phi}^{r} \Rightarrow \phi^{-r}=\bar{\phi}^{r}
$$

which means that $\bar{\phi}^{r}+\bar{\phi}^{-r}=\phi^{r}+\phi^{-r}$ and completes the statement of the theorem.

Theorem 3. For the Pell sequence $\left(P_{n}\right)_{n \geq 0}$ and $r \in \mathbb{Z}, r \equiv 0(\bmod 2)$

$$
P_{r+1}+P_{r-1}=\varphi^{r}+\varphi^{-r} .
$$

Proof. When applying (11) to the relation (20) we obtain

$$
\left[\frac{\varphi^{r+1}-\bar{\varphi}^{r+1}}{\varphi-\bar{\varphi}}+\frac{\varphi^{r-1}-\bar{\varphi}^{r-1}}{\varphi-\bar{\varphi}}\right]=\frac{\varphi^{n+r}-\bar{\varphi}^{n-r}+\varphi^{n-r}-\bar{\varphi}^{n-r}}{\varphi^{n}-\bar{\varphi}^{n}}
$$

which means that in order to prove the theorem we have to show that equality

$$
\frac{\varphi^{n+r}-\bar{\varphi}^{n+r}+\varphi^{n-r}-\bar{\varphi}^{n-r}}{\varphi^{n}-\bar{\varphi}^{n}}=\varphi^{r}+\varphi^{-r}
$$

holds true. Comparison of the l.h.s. and the r.h.s. of this relation gives

$$
\begin{aligned}
-\bar{\varphi}^{n+r}-\bar{\varphi}^{n-r} & =-\varphi^{r} \bar{\varphi}^{n}-\bar{\varphi}^{n} \varphi^{-r} \\
-\bar{\varphi}^{n}\left(\bar{\varphi}^{r}+\bar{\varphi}^{-r}\right) & =-\bar{\varphi}^{n}\left(\varphi^{r}+\varphi^{-r}\right) \\
\varphi^{-r}+\varphi^{r} & =\varphi^{r}+\varphi^{-r} .
\end{aligned}
$$

Finally, we employ a property that relates the silver ratio and its conjugate

$$
-\frac{1}{1+\sqrt{2}}=1-\sqrt{2}
$$

to show that above relation holds true. This completes the statement of the theorem. 


\section{Some Further Identities}

Using Theorem 1 and Lemma 2 various other identities can be proved. Some of them for the Fibonacci sequence are

$$
\begin{aligned}
8 F_{n} & =F_{n+4}+F_{n}+F_{n-4}, \quad n \geq 4 \\
9 F_{n} & =F_{n+4}+F_{n+1}+F_{n-2}+F_{n-4}, \quad n \geq 4 \\
57 F_{n} & =F_{n+8}+F_{n+4}+F_{n+2}+F_{n-2}+F_{n-4}+F_{n-8}, \quad n \geq 8
\end{aligned}
$$

and some of them for the Pell numbers are

$$
\begin{aligned}
3 P_{n} & =P_{n+1}+P_{n-1}+P_{n-2}, \quad n \geq 2 \\
4 P_{n} & =P_{n+1}+P_{n-1}+P_{n-2}, \quad n \geq 2 \\
20 P_{n} & =P_{n+3}+P_{n+2}+P_{n-3}+P_{n-4}, \quad n \geq 4 \\
40 P_{n} & =P_{n+4}+P_{n+2}+P_{n-2}+P_{n-4}, \quad n \geq 4 .
\end{aligned}
$$

For the purpose to prove identity (32) we have

$$
\begin{aligned}
& P_{n+3}+P_{n+2}+P_{n-3}+P_{n-4} \\
= & P_{n-1} P_{3}+P_{n} P_{4}+P_{n-1} P_{2}+P_{n} P_{3}+P_{n-1} P_{-3} \\
& \quad+P_{n} P_{-2}+P_{n-1} P_{-4}+P_{n} P_{-3} \\
= & 5 P_{n-1}+12 P_{n}+2 P_{n-1}+5 P_{n}+5 P_{n-1}-2 P_{n}-12 P_{n-1}+5 P_{n} \\
= & 20 P_{n} .
\end{aligned}
$$

Such identities can also be proved combinatorially, combining arguments presented in proofs of Lemma 1 and identity (21).

\section{References}

[1] M. Bicknell, A primer on the Pell sequence and related sequences, The Fibonacci Quart., 13, No 4 (1975), 345-349.

[2] A.T. Benjamin and J.J. Quinn, Proofs that Really Count, The Mathematical Association of America, Washington (2003).

[3] Jhon J. Bravo et al., Powers in products of terms of Pell's and Pell-Lucas Sequences, Int. J. Number Theory, 11 (2015), 1259-1274.

[4] A. Dujella, A problem of Diophantus and Pell numbers, In: Application of Fibonacci Numbers, Vol. 7 (Eds. G.E. Bergum, A.N. Philippou, A.F. Horadam), Kluwer, Dordrecht (1998), 61-68. 
[5] A.F. Horadam, Zeckendorf representations of positive and negative integers by Pell numbers, In: Application of Fibonacci Numbers, Vol. 5 (Eds. G.E. Bergum, A.N. Philippou, A.F. Horadam), Kluwer, Dordrecht (1993), 305316.

[6] A.F. Horadam, Maximal representations of positive integers by Pell numbers, Fibonacci Quart., 32, No 3 (1994), 240-244.

[7] D.E. Knuth, Fibonacci multiplication, Appl. Math. Lett., 1, No 1 (1988), $57-60$.

[8] S. Vajda, Fibonacci and Lucas Numbers, and the Golden Section: Theory and Applications, John Wiley and Sons, Inc., New York, 1989.

[9] E. Zeckendorf, Représentation des nombres naturels par une somme de nombres de Fibonacci ou de nombres de Lucas, Bull. Soc. R. Sci. Liege, 41 (1972), 179-182.

[10] E. Zeckendorf, A generalized Fibonacci numeration, The Fibonacci Quart., 10, No 4 (1972), 365-372. 
